\title{
Algunos puntos de partida de Compañeros de viaje: la lectura de Cántico en Jaime Gil de Biedma ${ }^{1}$
}

\author{
Daniel Arturo SAMPerio JiméneZ \\ El Colegio de México
}

Resumen: Mediante una revisión de Cántico: el mundo y la poesía de Jorge Guillén de Jaime Gil de Biedma, en este artículo se trata de entender algunos puntos de partida de Compañeros de viaje, poemario de Gil de Biedma que, si bien es un año anterior, se elaboró paralelamente al estudio sobre Guillén. Con ello se pretende entender por qué se trata de puntos de partida de su poética y comprender el decisivo sentido del relevo generacional que hay entre uno y otro poeta.

Palabras clave: Gil de Biedma, Guillén, lectura, objetividad, comunicación.

Some starting points of Compañeros de viaje: the lecture of Cántico in Jaime Gil de Biedma

Abstract: By a review of Cántico: el mundo y la poesía de Jorge Guillén of Jaime Gil de Biedma, this article is about understanding some starting points of Compañeros de viaje, book of poems by Gil de Biedma that, although it's a year before, was developed in parallel with the study on Guillén. We try to understand why these are starting points of his poetics and to understand the meaning of the decisive generational change between one poet and another.

Keywords: Gil de Biedma, Guillén, lecture, objectivity, communication.

1 A James Valender, con mucha gratitud. 


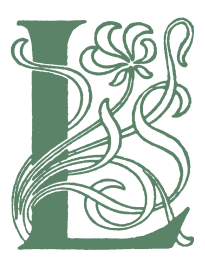

a publicación en 1960 de Cántico: el mundo y la poesía de Jorge Guillén por parte de Jaime Gil de Biedma supuso el abierto reconocimiento de este a la obra del poeta de la generación del 27. Publicado un año después del primer poemario en forma de Gil de Biedma, representó y reafirmó también el espacio que su obra quería ocupar en el panorama de la poesía española de la segunda mitad del siglo XX, hasta donde la tradición simbolista había dejado profundas huellas. El estudio, aparte de mostrar admiración por la obra de Guillén, delataba la influencia más fuerte que había en ese momento en la formación de Gil de Biedma como poeta.

Jorge Guillén fue uno de los poetas que Gil de Biedma más leyó en los años cincuenta, que puede considerarse la década formativa del poeta ${ }^{2}$. El estudio, como él mismo lo deja ver en sus páginas, fue una especie de "precipitación» de todas esas lecturas de Guillén. Esa serie de sucesivas experiencias lectoras fueron las que al final lo constituyeron. Aparte de los valiosos comentarios que Cántico merece a Gil de Biedma, el estudio es estimable porque permite ver otra faceta del poeta. Ésta es su faceta de lector. Un aspecto bastante interesante, a decir de la modestia que Gil de Biedma muestra en él, la cual - como en su poesía - vuelve a su trabajo más objetivo y honesto.

En un encuentro que tuvo lugar en la Universidad de Granada, en diciembre de 1983, Jaime Gil de Biedma se ocupó del tema de su experiencia como lector de poesía. Se trató de dos conferencias, cuya primera sesión tocó este asunto, mientras la segunda versó, en este caso, no tanto acerca de su experiencia personal en el quehacer poético, como sí acerca de la actividad del poeta moderno. Volvió a comentar en esta segunda sesión sus ideas sobre la poesía de la experiencia y planteamientos, cercanos a su poética, de The Poetry of Experience. The Dramatic Monologue in Modern Literary Tradition (1957) de Robert Langbaum, libro que ya había mencionado en el ensayo sobre Luis Cernuda, «Como en sí mismo, al fin», de la década anterior.

Sin embargo, en esta ocasión no son estas ideas las que se quisieran comentar aquí, sino las que tienen que ver con la experiencia de Jaime Gil de Biedma como lector. En aquella primera conferencia, el autor de Compañeros

2 La relación entre ambos poetas, si bien al principio estuvo marcada por una gran admiración, se enfrió en los últimos años. Sobre este respecto, véase el trabajo de Díaz de Castro (1996). 
de viaje se presentó ante todo como un mero lector de poesía: «Quisiera hablar de poesía un poco como se habla entre lectores, es decir, no hablar como crítico sino como lector» (Gil de Biedma, 2010: 1123). Un lector que habla de poemas entre cofrades y no desde la posición del crítico. Esto, por una parte, lo hizo alejarse de cierta utilización de términos de crítica literaria, términos abstractos a su parecer, y, por otra parte, lo llevó a tratar el tema de la poesía desde la perspectiva del creador. Esto resulta especialmente clave para Gil de Biedma, dado el valor que asigna a la lectura de poemas. Como lo entiende el poeta, esta actividad es más importante que el hecho de escribirla, puesto que la lectura de poesía representa una condición favorable para emprender la escritura de un poema. Es así que Gil de Biedma confiesa la razón por la que ha organizado sus conferencias empezando por la cuestión de la lectura de poesía. El creador se aproxima de esta manera a la poesía, pues la experiencia lectora ante esta expresión artística no representa para él una serie de valoraciones y juicios sobre determinadas obras, sino una posibilidad de reflexión acerca del propio trabajo creativo.

En este sentido, la actitud de Gil de Biedma es similar a la del crítico que había encontrado en T. S. Eliot al prologar su traducción de Función de la crítica y función de la poesía:

Eliot - a pesar de su no desdeñable erudición histórica y filosófica - se acerca a la actividad crítica a título de practicante del arte poética y gustador de poemas; se propone, antes que descubrir la esencia última de lo poético - si es que existe - hallar orientación para quienes, como él, escriben y leen poemas (Gil de Biedma, 1980: 22).

En su experiencia personal, Gil de Biedma encuentra que haber leído poesía ha sido más importante que escribirla. Esto es, «mucho más determinante y mucho más condicionante» (Gil de Biedma: 2010: 1124). Ello hasta tal punto de que el poeta se podría imaginar a sí mismo, tal cual era en ese momento, sin haber escrito un solo poema. Al decir que la lectura ha sido más decisiva, Gil de Biedma no deja de referirse a que es por ese acto de retroalimentación de la lectura que ha escrito poemas. Para él, «escribir poemas está en función de haberlos leído y de esperar seguir leyéndolos» (Gil de Biedma: 2010: 1129). El poeta llega a esa conclusión tras haber reflexionado acerca de cómo el poema se inscribe dentro de otros poemas para introducir una pequeña diferencia significativa que lo distinga. Esto hace pensar de nuevo 
en la presencia de Eliot en las reflexiones de Gil de Biedma, en este caso, por aquel otro célebre ensayo del autor de Four Quartets, quien en «La tradición clásica y el talento individual» afirma: «Los monumentos existentes conforman un orden ideal entre sí, que se modifica por la introducción de la nueva obra de arte (verdaderamente nueva) entre ellos» (Eliot, 2000: 19-20). Es por medio de la lectura que Gil de Biedma sitúa el quehacer poético, en tanto que el poema que se escribe se sabe de antemano en relación y diálogo con otros tantos poemas leídos.

El papel de la lectura no es sólo más importante que el de la escritura de poesía, dado que la determina, sino que se trata de un acto que en sí constituye la existencia real de la poesía: «Creo que la poesía es una resultante del poema, es decir, que un poema mientras uno lo escribe no es poesía. [...] Poesía es ese poema leído por alguien.» (Gil de Biedma, 2010: 11321133).

Gil de Biedma está en una postura que refleja cierta idea sobre la voluntad del lector en la participación de la emoción del poema ${ }^{3}$. Una cuestión de la que ya se había ocupado en el prólogo de Función de la crítica y función de la poesía, donde hacia el final trató el asunto de la poesía como comunicación ${ }^{4}$. En este prólogo, después de que Gil de Biedma reconoce la diferencia entre transmisión y comunicación, encuentra modos de entender esta última. Entre éstos, le parece más genuino aquel que se establece no de hombre a hombre, sino de poeta a lector por mediación del poema. Esto, cuando se ha entendido que no es la emoción la que suscita el poema sino viceversa, en tanto que el poema trata con materiales latentes en la vivencia del poeta, a los que «trae a la conciencia, los polariza y les confiere un valor operacional que los hace inteligibles» (Gil de Biedma, 1980: 28). El acto de lectura supone,

\footnotetext{
3 No se puede olvidar que por los mismos años en que aparece el estudio de Gil de Biedma, se había publicado La hora del lector de J. M. Castellet, una obra precursora de la teoría de la recepción y del importante papel rol del lector en la obra.

4 Poesía como comunicación o como conocimiento, un tema especialmente espinoso en la poesía española de esos años como lo muestran textos del tipo de «Poesía no es comunicación» de Carlos Barral o Teoría de la expresión poética de Carlos Bousoño. Gil de Biedma no estuvo al margen de la polémica. Evitando que se convirtiera en una polémica estéril, anotaba con natural perspicacia observaciones como la siguiente: «Poesía es comunicación, porque el poema hace entrar a su autor en comunicación consigo mismo. O quizá: poesía es conocimiento, según asegura otra ecuación bastante frecuente y cuyo preciso importe me parece igual de incalculable; al fin y al cabo, en el juego de reducir un término abstracto a otro término abstracto, no apostamos a otra ganancia que a la de las posibilidades metafóricas de entrambos, y las metáforas posibles son muchas» (Gil de Biedma, 1980: 28-29).
} 
entonces, que el lector entra en comunicación con esa vivencia polarizada por la escritura del poema; pero también Gil de Biedma concibe otro modo de comunicación, por el cual «autor y lector, cada uno por separado, se enfrentan con el poema y entran en comunicación consigo mismos» (Gil de Biedma, 1980: 29). En este caso, ya no sigue teniendo la misma importancia la comunicación de la vivencia (polarizada por la escritura) desde la experiencia del poeta a la del lector por medio del poema, sino independientemente ambos con respecto a este. De este modo la voluntad del lector es significativa, pues su participación implica que es él quien, desde su particular punto de vista, crea el poema: «El acto de la lectura es también un acto creador, y la emoción poética puede tener para el lector un valor muy distinto del que tuvo para el poeta» (Gil de Biedma, 1980: 29).

Desde la perspectiva del autor de Moralidades, el significativo peso que recae en la voluntad del lector plantea uno de los problemas de la poesía, que radica en el hecho de que «el lector alcance el suficiente grado de seguridad en sí mismo para no preocuparse de si entiende o no entiende el poema» (Gil de Biedma, 2010: 1138). Para ello, el lector requiere cierta pasividad activa: «Realmente, por ejemplo, leer poesía consiste en una pasividad activa, en que uno tiene que estar empleando un esfuerzo, una disciplina mental bastante sólida en mantenerse absolutamente pasivo.» (Gil de Biedma, 2010: 1138).

Una de las lecturas más manifiestamente presentes en Jaime Gil de Biedma fue la de la obra de Jorge Guillén. Entre sus lecturas de juventud, Cántico se contaba junto con la de los poetas del Siglo de Oro, los otros poetas de la generación del 27, Baudelaire, Mallarmé, Rimbaud, Valéry, los surrealistas y Rilke. Pero con Guillén se trataba de una lectura especial, como afirma en el texto «La imitación como mediación, o de mi Edad Media»: «Mi maestro absoluto era Jorge Guillén, el Guillén de las tres primeras ediciones de Cántico, con quien yo aprendí el arte de hacer poemas» (Gil de Biedma, 2010: 1066). Esto ya habla de una lectura dirigida y de ninguna manera desinteresada. El acercamiento a la obra inicial de Guillén representó para Gil de Biedma el aprendizaje consciente del oficio de poeta. Este refiere su primer contacto con Cántico en «Historia de una experiencia literaria», el capítulo inicial de su estudio sobre la poesía de Jorge Guillén. 
Hay una serie de circunstancias que habría que considerar en ese primer acercamiento de Gil de Biedma a Guillén. Por una parte, el hecho de que fue la edición de 1945, es decir, la tercera, la que en un principio leyó. Por otra, la eventualidad de haberse encontrado con ese poemario en cierta etapa de la vida en que se hallaba, cuando hacia la década de los años cincuenta era un muchacho de más de veinte años que, como algunos muchachos de su edad, tenía frecuentemente presente una palabra que «repetía como una especie de conjuro: objetividad» (Gil de Biedma, 1960: 14). Palabra que para un poeta en ciernes resultaba fundamental, pues se convertía en «el sésamo maravilloso que le hará a uno salir de sí mismo. ¡Con qué alegría se descubre que, por mal que uno ande, hay cosas en este mundo que están francamente bien!» (Gil de Biedma, 1960: 14). Para este joven poeta, Cántico era como una válvula de escape por donde podía respirar una bocanada de aire fresco. Así confiesa el poeta algunos años después en el libro, donde iba a volcar parte de esa experiencia, que, cuando presentía que iba perdiendo el sentido de las cosas o que iba a caer irremediablemente dentro de sí mismo, tomaba de inmediato el volumen de Cántico.

La primera circunstancia habría que comentarla suficientemente, pues representa una contingencia significativa en la formación de Gil de Biedma como poeta. Entre la segunda edición de Cántico. Fe de vida (Cruz y Raya, Madrid, 1936) y la tercera (Litoral, México, 1945) mediaba la experiencia de la guerra civil española. Lo que Gil de Biedma encontró en esta tercera edición fue clave:

Era la apasionada reacción de los años posteriores a la guerra, el terco rehusarse al propio dolor y a la propia angustia, en un intento por salvar la realidad del mundo, lo que a mí me interesaba entonces y lo que yo podía comprender, y no me importaba tanto la jubilosa realidad del mundo como el empeño decidido de afirmarla por encima de uno mismo, casi trágicamente (Gil de Biedma, 1960: 16).

Una contingencia que de haberla encontrado Gil de Biedma distinta - haberse encontrado con la primera o segunda edición de Cántico-, no hubiese producido el mismo efecto. De ese modo, no habría tenido la experiencia de lectura de Cántico esa conjunción a la que Gil de Biedma se refiere. 
Esa lectura queda reflejada en el estudio sobre el primer poemario de Jorge Guillén. Se puede ver en qué sentido esa palabra tan recurrente, la objetividad, daba vueltas en la mente de aquel joven poeta. La lectura de la poesía de Guillén le parecía clarificar el alcance de esa palabra a la que acudía como conjuro. El segundo capítulo del estudio es elocuente de ello. En «Cerco del presente», Gil de Biedma se detiene en una serie de aspectos del quehacer poético, que especialmente tienen que ver con el aquí y el ahora. En la primera sección del capítulo, Gil de Biedma se asombra de la cantidad de cosas que aparecen en la poesía de Guillén, cuando «la poesía lírica ha mostrado desde siempre una cierta tendencia a generalizar sus asuntos y a rehuir el trato con los objetos de uso diario» (Gil de Biedma, 1960: 26). Una presencia de cosas y objetos en una poesía como tal lleva a Gil de Biedma a concebir en Cántico un gusto de su autor por motivos y temas de la vida diaria. De ahí que «el protagonista de esta poesía no es tanto el individuo como la persona» (Gil de Biedma, 1960: 27): un ser más concreto y perfectamente situado en un aquí y un ahora; sobre todo, un ser que en su existencia participa de sus sentidos más que de su conciencia de individuo. Se trata de alguien «inmediato a las cosas» (Gil de Biedma, 1960: 28). De este modo se tienen dos elementos eje sobre los que se coordina la poesía de Guillén, a decir de la lectura de Gil de Biedma. Por un lado, las cosas, objetos que existen en diversidad y que provienen todos de la vida diaria, en la que se sitúa esa poesía, y, por otro, la presencia de un sujeto que ante todo es persona, cuyos sentidos sensoriales participan de la inmediatez: «El hombre está inmediato a las cosas. Inmediatez es el término que mejor define esa vertiente elemental del mundo de Guillén» (Gil de Biedma, 1960: 28).

Sin embargo, del otro lado de aquello que cabe en la inmediatez, se encuentra el otro término del mundo poético de Guillén: la reflexión. Gil de Biedma la imagina coexistiendo con la inmediatez a tal grado que «son los dos polos que simultáneamente juegan para definir la actitud del protagonista de Cántico con respecto a la realidad exterior» (Gil de Biedma, 1960: 29). Se puede ver Cántico como la superposición de dos planos que permiten reparar en la profundidad del mundo. Además, la inmediatez y la reflexión plantean una especie de coexistencia necesaria, ya que sin el punto de alejamiento de la reflexión, el deslumbramiento inmediato de las cosas cegaría. Gil de Biedma identifica estos polos como una dialéctica de la discursividad del poema, pero no tanto porque así estén dados por el poeta, Guillén, sino porque de 
esta manera los interpreta el autor del estudio. De ahí que «la lectura de un poema de Cántico exige constantes modificaciones y reajustes del punto de vista en que el lector se sitúa» (Gil de Biedma, 1960: 31). De esta manera al poema se añade un tono, que está determinado por cada uno de estos dos puntos de vista y actitudes mentales, ya sea de inmediatez o reflexión, que se adoptan durante el proceso del discurso. Este tono revela, aparte, dos tipos de actitudes en que oscila la relación del sujeto con las cosas:

Esas bruscas transiciones, ese juego simultáneo de dos disposiciones opuestas - resueltas siempre en la unidad del poema - responden a algo más que a un simple afán de primores estilísticos: son la traducción poética de una actitud humana que está en la base de todas nuestras relaciones con la realidad (Gil de Biedma, 1960: 31).

Dentro de la concepción de Gil de Biedma de que el sujeto de la poesía de Guillén se halla en medio de una espesa red de relaciones, hay además esa clase de matices que hacen del mundo poético de Cántico una experiencia enriquecedora. Ello no solamente en el sentido del feliz encuentro entre sujeto y mundo, sino por la complejidad de ese encuentro y su paulatino descubrimiento. Algo que ciertamente acentúa aún más uno de los principales valores que Gil de Biedma observa en Cántico.

La relación constante entre el sujeto y las cosas hace que la soledad más terrible - la idea de que las cosas son prolongación de la individualidad y, por ende, que el sujeto está absolutamente solo en el universo- es casi desconocida en la poesía de Guillén. Ese sujeto, que es persona más que individuo, dado que está supeditado a una serie de relaciones, no padece la soledad. No sólo es acompañado por las cosas, que para entrar en relación con el sujeto deben mantener su otredad, sino que es determinado por éstas y de ellas toma dominio, pues: «Las cosas son definitorias: sitúan al hombre, y el hombre al situarse se entraña, toma posesión de sí mismo: gracias a que sabe qué hace y dónde está sabe quién es» (Gil de Biedma, 1960: 35).

El tema del ser en Guillén también consta en la lectura de Gil de Biedma, tema que la mayoría de la crítica, según lo deja ver este, no lo consideraba sin antes teñirlo de preocupaciones ontológicas; con pretensiones filosóficas de «poeta abstracto, poeta esencial» (Gil de Biedma, 1960: 38), algunos críticos adjudicaban ciertas etiquetas a Guillén. Para Gil de Biedma, en cambio, lo 
que queda bastante claro, tras una lectura sin pretensiones filosóficas de Guillén, es que sencillamente este halla un regocijo de ser y que en sí esta palabra tiene un lugar de privilegio en el vocabulario del poeta. La diferencia fundamental, entonces, con respecto a esas otras lecturas filosóficas de Guillén residiría en que puesto que este «no parte de un concepto, sino del hecho de ser y de sentirse ser, la ambigüedad que trae aparejada la formulación idiomática de su pensamiento constituye la raíz misma de su visión poética, lo que hace de ella algo emocionante y vivo» (Gil de Biedma, 1960: 39). Ahora bien, ese sentimiento de ser ha de traer aparejada su conciencia. Es lo que Gil de Biedma encuentra inevitablemente tras el maravillado asombro ante el reconocimiento y la verificación de las cosas circundantes. Se trata de una conciencia que es capaz de articular y pronunciar un yo. No solamente es el regocijo de ser, sino que también implica el «gozo de ser un ser determinado, una concreta persona con cédula y filiación, nacida en un lugar y en unas circunstancias únicas» (Gil de Biedma, 1960: 43). Se puede ver un tránsito que va del gozo de sencillamente ser al regocijo de saberse alguien.

Con Cántico: el mundo y la poesía de Jorge Guillén, Jaime Gil de Biedma abrió sin duda un espacio para escribir su propia poesía. El interés del estudio trasciende lo que Gil de Biedma diga de Cántico, puesto que en él «vemos reflejado el diálogo que a lo largo de los años cincuenta el autor de Compañeros de viaje entablara consigo mismo en su esfuerzo por superar la tradición simbolista» (Valender, 2010: 48). Términos que forman parte del vocabulario de la poética de Guillén como la objetividad, la inmediatez, la reflexión, el ser, todos ellos ayudaron sin duda alguna a superar esa tradición. El hecho de que Gil de Biedma partiera de ellos para su estudio es sintomático de en qué medida representaron importantes conceptos para él y su quehacer poético. En ese sentido, éstos significaron puntos de partida para el poeta que publica su primer libro en forma por esos años. Pienso que para una lectura de Compañeros de viaje resultan útiles para abrir perspectivas de estudio no sólo de los poemas de la colección, sino también de la reflexión poética de un autor que busca un lugar desde el cual situar su poesía.

Hay algunos riesgos en hacer este trasvase de los ensayos del autor a su poética y de ésta a la poesía, aunque tal metodología no deje de contar con aspectos a su favor ${ }^{5}$. Sin embargo, no es ésta la intención de este artículo. Lo

5 «A lo que lleva finalmente esta metodología es a limitar nuestra percepción de los poemas del autor a aquellos elementos previstos por su poética. Desde luego, Gil de Biedma es un poeta sumamente cons- 
que menos se pretende es hacer una lectura reduccionista de tales poemas a la luz de únicamente lo que permita ver la lectura de Guillén por medio del libro sobre Cántico. El objeto de estudio, en este caso, no son tanto los poemas como la reflexión sobre los puntos de partida con los que contó Gil de Biedma para la elaboración del poemario de 1959 y, con base en esto, comprender el decisivo sentido del relevo generacional que hay entre uno y otro poeta.

Para empezar habría que retomar el hecho de la lectura de la tercera edición de Cántico, por parte de Gil de Biedma hacia los años cincuenta, para situar un contexto donde la palabra objetividad representaba una especie de conjuro para él y para algunos de su generación. No habría que partir de este primer término del mundo poético de Guillén, sin dejar de tomar en cuenta el sentido y repercusión que este pudo tener en un contexto como aquél. De este primer término se desprenden con naturalidad los demás, como en el poema que abre la sección «Por vivir aquí» de Compañeros de viaje. En «Arte poética» se observa la voluntad del sujeto del poema por ser y por vivir en la inmediatez de la experiencia:

Es sin duda el momento de pensar

Que el hecho de estar vivo exige algo,

Acaso heroicidades - o basta, simplemente,

Alguna humilde cosa común

Cuya corteza de materia terrestre

Tratar entre los dedos, con un poco de fe? (vv. 13-18)

Sin embargo, estos términos resuenan en el poema de Gil de Biedma no sin antes adquirir y proyectar otros sentidos. En los demás poemas de la sección es evidente la presencia de un sujeto y de una voz yo, pero un sujeto que adopta la postura de la reflexión, más que la de la inmediatez. En lugar de tratarse de un sujeto rodeado por cosas que lo sitúan, se encuentra rodea-

ciente de lo que hace a la hora de construir sus poemas, así que las sugerencias que nos hace en cuanto a su lectura suelen resultar reveladoras. Y, sin embargo, como él mismo nos recordó más de una vez, un poema es algo más que la puesta en práctica, algo mecánica, de una serie de presuposiciones, sean de orden estético o afectivo. Así, al querer buscar en la poesía de Gil de Biedma una simple comprobación de sus teorías poéticas, la crítica corre el riesgo de pasar por alto lo específico de cada texto, ese movimiento espontáneo e imprevisto que, dentro de los parámetros establecidos, le confiere su vitalidad y su gracia.» (Valender, 2010: 50).

6 Todos los poemas se citan de Las personas del verbo (Galaxia Gutenberg, Barcelona, 2012), que reúne la poesía completa de Jaime Gil de Biedma. 
do de tiempo y relaciones humanas. Una situación que lo hace adoptar una actitud reflexiva. El par de versos iniciales de «Idilio en el café» son más que elocuentes: «Ahora me pregunto si es que toda la vida / hemos estado aquí. [...]». Desde el comienzo, el tono reflexivo impregna el poema. Incluso cuando aparece una sensación de inmediatez, ésta no deja de estar impregnada por este tono de extrañamiento: «[...] Pongo, ahora mismo, / la mano ante los ojos - qué latido / de la sangre en los párpados - [...]» (vv. 2-4). Pero el poema no queda solamente ahí con los términos de reflexión y persona de los que parte, sino que se proyecta en nuevas dimensiones de un mundo poético, del que los anteriores términos han sentado las bases. Si el sujeto se sabe situado en un café y rodeado de los factores del tiempo, los cuales lo colocan en una actitud meditabunda, entonces se proyecta también situado en relación con los otros. No por otra situación se pueden entender los siguientes versos que prolongan la actitud reflexiva del sujeto: «[...] ¿Quiénes son, / rostros vagos nadando como en un agua pálida, / éstos aquí sentados, con nosotros vivientes?» (vv. 7-9).

La relación del sujeto con el tiempo, en específico, el pasado, representa otra proyección de los términos presentes en el mundo poético de Guillén. Proyección que está presente en el grupo de poemas de «Por vivir aquí» que versan en general sobre el recuerdo. En ellos habita un sujeto situado en el tiempo, que por ese solo hecho se encuentra sometido a una serie de recuerdos, que en el caso de cada poema adquieren un valor especial. "Aunque sea un instante» plantea la existencia del sujeto en medio de una realidad a la que en el fondo teme. Por ello este invoca el pasado como un paliativo, el cual, sin importar si realmente existió tal como lo recuerda el sujeto, le haga creer que al menos ha vivido. Es como si la plenitud de ser irradiara sus luces desde ese pasado. «Recuerda» también supone el ser en el pasado: «Hermosa vida que pasó y parece / ya no pasar...» (vv. 1-2). Como en el poema anterior, desde un preciso instante se hace este llamado o irrumpe el pasado en la discursividad de un sujeto situado en el tiempo. No hay en este sentido inmediatez, pues las relaciones con el mundo no son vivas, sino que están canceladas por esa relación del sujeto con el tiempo. El recuerdo ya no aparece asociado con otro tiempo, que se invoca por estar pleno de ser y de vida, sino con su contrario, la muerte. Es el caso de «Al final», en donde el recuerdo constante de una imagen desvanecida conduce al sujeto, tras muchas vueltas, a encontrarse realmente muerto. Con «Muere Eusebio» y «Noches 
del mes de junio», el recuerdo aparece fijado en momentos precisos del pasado del sujeto. Recuerdos de infancia y de adolescencia, respectivamente, sitúan al sujeto como persona enclavada en una circunstancia determinada: «-nuestra infancia en los años de la guerra civil» («Muere Eusebio», v. 20) $\mathrm{y}$ «ciertas noches de junio de aquel año, / casi borrosas, de mi adolescencia / (era en mil novecientos me parece / cuarenta y nueve)» («Noches del mes de junio», vv. 2-5). La postura reflexiva priva en estos poemas y, sobre todo, va discurriendo tentativamente hacia el encuentro del sujeto con los objetos de un pasado propio, a la vez impreciso y hondo. En «Vals del aniversario» el sujeto no rememora su pasado, sino que pareciera que ocurre una actualización de este. El pasado se actualiza con el aniversario no sin dejar una sensación de malestar: «Ha oscurecido el cuarto / mientras que nos miramos tiernamente, incómodos / de no sentir el peso de tres años» (vv. 14-16). La conciencia del sujeto, perfectamente identificable en las circunstancias que lo rodean, aparece sobre todo en «Infancia y confesiones». El sujeto se describe entre las circunstancias de su llegada a la ciudad, su origen familiar, su infancia. Pareciera que el sujeto del poema es consciente de lo inusual que es presentar de esta manera el yo y muestra un poco de pudor: «Yo nací (perdonadme) / en la edad de la pérgola y el tenis» (vv. 22-23). Después de hablar de ese mundo de la infancia, el sujeto sitúa parte de ese pasado en el presente en que vive:

De mi pequeño reino afortunado me quedó esta costumbre de calor y una imposible propensión al mito. (vv. 35-37)

La serie de poemas «Sábado», «Domingo» y «Lunes» son los más paradigmáticos de la sección en presentar esa relación del sujeto con el tiempo, dentro del cual este se inscribe. Pero en este caso, no con el tiempo pasado sino con el presente. En medio de ese tránsito de tiempo actual hay lugar para la inmediatez («Toca - para creer - la piedra / mansa, la paciencia del pretil», «Sábado», vv. 7-8), con lo cual se vuelve más intensa la relación. El hecho de que los poemas estén ordenados en sucesión de esos tres días sitúa al sujeto en un presente que es compartido por muchos hombres: el tiempo que se mide en la alternancia entre fines de semana y días laborables.

Esta presencia del sujeto en un tiempo que transcurre tal cual en la vida diaria reaparece en «De ahora en adelante», el poema con que se cierra la 
sección «Por vivir aquí». Hay en esta composición una especie de perplejidad del sujeto por hallarse en un tiempo que es nuevo para él. Después del extrañamiento, el sujeto lo reconoce:

Pero ya desde ahora siempre será lo mismo.

Porque de pronto el tiempo se ha colmado

y no da para más. Cada mañana

trae, como dice Auden, verbos irregulares

que es preciso aprender, $[\ldots]$

Como reconoce también, en ese tiempo nuevo está la presencia de los otros, con quienes cuenta, lo mismo que ellos cuentan con él. Esta presencia de los otros aparece inmejorablemente en el poema que abre el libro. "Amistad a lo largo» no sólo concibe a los otros en su discursividad, sino que está dirigido a ellos, quienes como el sujeto del poema están insertos en un tiempo: «Pasan lentos los días / y muchas veces estuvimos solos. / Pero luego hay momentos felices / para dejarse ser en amistad» (vv. 1-4). El poema, aunque formulado en tono reflexivo, no deja de mostrar el azoro que vive el yo ante ese reconocimiento, que no es como en Guillén una toma de conciencia de las cosas, sino de las personas: «Mirad: / somos nosotros» (vv. 5-6). La proyección de la red de relaciones del sujeto con las cosas resuena aquí en tanto vinculación con otros tantos sujetos, en medio de un tiempo y una historia que les pertenece.

El carácter de estos poemas es paradójico con respecto al título de la sección que los reúne. El vivir aquí no alude a la plenitud de ser, sino a todas las muy diversas implicaciones de estar aquí: un estar con toda su carga de recuerdos y esperanzas, con sus percepciones del mundo inmediato; es decir, la propia identidad.

Como se puede ver, los puntos de partida inferidos del estudio sobre Cántico de Gil de Biedma (objetividad, afirmación de la realidad del mundo, inmediatez, reflexión, el sujeto) aparecen como términos que resuenan en los poemas de Compañeros de viaje; sin embargo, lo hacen adquiriendo sentidos propios. Por un lado, en los poemas de Gil de Biedma pesa más la reflexión que la inmediatez como se puede constatar por la postura que adopta el sujeto. Por el otro, hay además en estos poemas un estar y saberse alguien por encima de un ser. Como se mencionó apenas, el vivir aquí no alude a una 
plenitud ontológica, sino a las derivaciones existenciales que suponen estar en un tiempo histórico y en la compañía humana: aquí, ahora y con los otros.

Estos dos elementos pueden objetivarse con sentidos propios en la poética de Gil de Biedma, quien ya se aleja de Guillén después de haber tenido en este su punto de partida. Hay con ello un importante relevo generacional entre ambos poetas, debido a que en líneas generales compete a un cambio de sensibilidad en la poesía española del siglo XX.

Por lo pronto, cabe partir del hecho de que el estudio sobre Cántico representó para su autor una forma de retribuir su admiración por la poesía de Guillén, pero también, y sobre todo, un medio para elaborar sus propias ideas alrededor de la poesía que buscaba hacer. Se trataba de la formulación de un discurso poético que se proponía la superación de la tradición simbolista, la cual se halla tan arraigada en la generación del 27. Para ello, la obra de Guillén supuso para Gil de Biedma una excelente vía de entrada. De ninguna manera esto pudo haber sido premeditado, sino producto de un complejo proceso. De otra forma, no sería tan interesante por qué precisamente el autor de Moralidades partió del mundo poético de Jorge Guillén.

Gil de Biedma siempre subrayó el aprendizaje consciente del oficio de poeta en su acercamiento a Cántico. Como se mencionó, en su momento leyó con mucha atención la edición de 1945. Cuando varios años después, en una conferencia, reafirma el valor de la poesía de Guillén, insiste en esa tercera edición por encima de sus siguientes poemarios ${ }^{7}$. Curiosamente, es la poesía de Guillén hasta esta fecha la que categóricamente siempre reconoció como la mejor, porque de hecho correspondía a la época en que se formaba como poeta. En ese momento, era un lector acucioso por aprender el trabajo de hacer poemas y, sobre todo, hacerlos como parte de una búsqueda creativa que se habría de clarificar con el paso del tiempo.

Por eso que resultara tan clave que haya partido de la poesía de Jorge Guillén. La faceta de Gil de Biedma como poeta lector, que se ha podido atisbar, permite explicitar la aparición de su primer libro de poemas en relación y

\footnotetext{
7 «Ahora, si Jorge Guillén es un gran poeta es por las tres primeras ediciones de Cántico. Si Cántico desapareciese o fuese olvidado, el resto de la obra de Guillén es la de un competente escritor de versos que, al menos para mí, no merece la calificación de gran poeta que por las tres primeras ediciones de Cántico le otorgamos.» (Gil de Biedma, 2010: 1029).
} 
diálogo especial con Cántico. Precisamente, no se trata de una mera emulación, sino de verdad de una lectura atenta de aquello que Gil de Biedma encontraba de más esencial en la poesía de Guillén para su propio trabajo. Es lo que se puede ver en los puntos de partida de Compañeros de viaje. Gil de Biedma toma elementos del mundo poético de Guillén y los re-significa para construir el suyo propio. Al final, esos elementos, diríamos puntos de partida, tienen que ver más con Gil de Biedma que con el propio Guillén, pero también serían impensables en la poesía del primero sin este. Su aparición en Gil de Biedma está ligada a un vivo diálogo con la obra poética del autor de Cántico.

Sin embargo, se trata de un diálogo que también expresa el sugerente relevo generacional que ocurre entre ambos poetas en el contexto de la poesía española de esos años. Al final del estudio sobre Cántico, libro que había llevado a Gil de Biedma varios años redactar entre altibajos ${ }^{8}$, hay un desencanto de la poesía de Guillén; particularmente, debido a que a su parecer y a pesar de la profunda admiración hacia su poesía, ésta representaba la última manifestación de la tradición simbolista. Es una especie de reproche que, en realidad, cabe entenderlo como un reproche de Gil de Biedma dirigido hacia él mismo, puesto que sentía que aquella debía ser ya una tradición superada en la poesía que se proponía escribir.

Significativamente, Gil de Biedma llegó a un punto en que, tras su enorme admiración, abjuró de la poesía de Jorge Guillén por su relación con la tradición simbolista. Pero, sin otro punto de partida que no fuera este, Gil de Biedma no habría podido formular un discurso poético propio. Esto tiene mucho que ver con el tipo de poesía que buscó escribir, cuya formulación definitiva halló con la lectura de la tradición poética anglosajona: se sabe que, en poetas como T.S. Eliot y sobre todo en W. H. Auden, dio con una actitud distinta ante la poesía «en la ambivalencia de la identidad, en esa doble conciencia que hace que me reconozca - simultáneamente o alternativamente- uno, unigénito, hijo de Dios, y uno entre otros tantos, un hijo de vecino» (Gil de Biedma, 2010: 806). Pues bien, en Guillén encontraría un germen incipiente que lo llevaría hacia nuevos derroteros no sólo para su propia obra sino para la poesía española de esos años.

8 En el volumen de Diarios (2015) se puede advertir que la redacción del estudio terminó pesando bastante en Gil de Biedma hasta llegar casi al hartazgo. 


\section{REFERENCIAS BIBLIOGRÁFICAS}

Barral, Carlos (1953), «Poesía no es comunicación», Laye, núm. 23, abril-junio, pp. 23-26.

Biedma, Jaime Gil de (1960), Cántico: el mundo y la poesía de Jorge Guillén, Seix Barral, Barcelona.

- (1980), «Función de la poesía y función de la crítica, por T. S. Eliot», en El pie de la letra. Ensayos 1955-1979, Crítica, Barcelona, pp. 17-31.

- (2010), «Como en sí mismo, al fin», «Lean ustedes a Jorge Guillén», «La imitación como mediación, o de mi Edad Media», «Leer poesía, escribir poesía», en Obras. Poesía y prosa, Galaxia Gutenberg, Barcelona, pp. 804820, 1028-1043, 1063-1081 y 1123-1183.

- (2012), Las personas del verbo, Galaxia Gutenberg, Barcelona.

- (2015), Diarios, Lumen, Barcelona.

Bousoño, Carlos (1970), Teoría de la expresión poética, 5a . ed., Gredos, Madrid.

Castellet, J. M. (1957), La hora del lector, Seix Barral, Barcelona.

Díaz de CAstro, Francisco J. (1996), «Lectura y escritura en Jaime Gil de Biedma», en Túa Blesa (ed.), Actas del Congreso Jaime Gil de Biedma y su generación poética, Gobierno de Aragón, Zaragoza.

ELIOT, T. S. (2000), «La tradición y el talento individual», en Ensayos escogidos, selección y prólogo Pura López Colomé, unAM, México, pp. 19-20.

VAlender, James (2010), «Introducción» a Obras. Poesía y prosa, Jaime Gil de Biedma, Galaxia Gutenberg, Barcelona, pp. 7-73. 